UDC 633.331

(C) 2016

V. Bulgakov, Academician of the National Academy of Sciences of Ukraine, Doctor of Technical Sciences

National University of Bioresources and Natural Resources of Ukraine

O. Adamchuk

State Enterprise "Scientific and Technical Center certification

"AgroSEPRO" NAAS

\title{
THEORETICAL STUDY OF CENTRIFUGAL FERTILIZER DISTRIBUTOR
}

The purpose. Determination of the optimum kinematic and design parameters of centrifugal fertilizer distributor. Methods. Methods of the theory of agricultural machines, theoretical mechanics, higher mathematics, and also creation of programs and numerical calculations for PC. Results. New differential equation of movement of a material corpuscle of artificial fertilizer along a blade of centrifugal end-effector of fertilizer distributor in view of an angle of slope of dispersing disk is created and its complete solution in the locked kind is given. Conclusions.

Graphical dependences of change of value of absolute velocity of tails of corpuscles of fertilizer from centrifugal scattering end-effector on value of an angle between its axis of rotation and vertical surface, and also changes of value of radiuses of feed of fertilizer and different angular velocities of rotary motion of dispersing disk are gained.

Key words: artificial fertilizer, centrifugal distributor, material corpuscle, movement, force, mathematical model, differential equation, absolute velocity.

Formulation of the problem. The efficiency of production of various crops depends to a large extent on the use of mineral fertilizers, which are most often introduced into the fields by surface method, spreading, using machines equipped with centrifugal scattering working organs. These working bodies, with undoubted advantages over other types of working bodies, however, require further improvement, concerning, first of all, the uniform distribution of fertilizers along the surface of the field. Our new designs of centrifugal scattering working bodies with rotation axes inclined 
at an angle to the horizontal plane, proposed by us, raise some performance indicators, but research and further search for their optimal constructive and kinematic parameters represent the actual scientific and technical task.

It is known that the working width of the machine grab for the introduction of mineral fertilizers in a centrifugal manner depends on the value of absolute velocity $V_{A C}$ Coming from fertilizer on the surface of its scattering working organ (disk) and $\alpha_{A C}$ the angle between its vector and the horizontal plane. Value $V_{A C}$ depends on the geometric parameters and kinematic modes of the work of the centrifugal scattering working organ, as well as on the physical and mechanical properties of mineral fertilizers.

As a result of the conducted research, the optimization of the geometric parameters of the centrifugal scattering working organ, taking into account the physical and mechanical properties of mineral fertilizers, was performed [1, 2]. It was found that an increase in the kinematic operating modes of the centrifugal scattering working body is limited to the strength of fertilizer granules. Therefore, with existing structural materials and types of fertilizers, the possibility of increasing the working width of the capture of machines by increasing the value $V_{A C}$ is exhausted.

When creating a centrifugal scattering working body with a sloping axis of rotation, it is necessary to have a methodology that would provide the definition of the absolute speed of the approach of fertilizers from its surface and the angle between the vector of the latter and the horizontal plane, depending on the parameters and operating modes of the centrifugal scattering working organ, as well as the physical and mechanical properties of the mineral fertilizers.

Analysis of recent research and publications. The author of the work [2] states that increasing the width of the absorption of mineral fertilizers by centrifugal dispersing working body is possible by achieving 
rational angular values $\alpha_{A C}$. The obtained results of the research indicate that rational values $\alpha_{A C}$ are in the range of $30 \ldots 35^{\circ}$. At the same time, the author of the work [3] has found that existing centrifugal scattering working bodies can achieve achievement value $\alpha_{A C}$ not more than $15.7^{\circ}$. Centrifugal scattering working bodies with a sloping axis of rotation ensure achievement of rational values $\alpha_{A C}$.

A well-known technique is the use of which one can determine the absolute speed of descent of fertilizer from a centrifugal scattering working body whose axis of rotation is vertical [4] and a method by which one can determine the absolute speed of the ascent of the fertilizer fraction from the centrifugal scattering working body whose axis of rotation is horizontal $[5,6$ ], unfortunately, do not consider the methods of determining the absolute speed of the fall of the fraction of fertilizers from the centrifugal scattering working body whose axis of rotation is sloping to the horizon the plane.

The aim of the study. Determination of optimal kinematic and structural parameters of a centrifugal mineral fertilizer spreader on the basis of obtaining new analytical dependences of the absolute rate of movement of mineral fertilizer particles at the moment of their ascent from a scattering disk, whose axis of rotation is located at an angle to the horizontal plane, and the blades are radially installed on its working surface..

Research methods. In carrying out analytical research methods of the theory of agricultural machines, theoretical mechanics, higher mathematics, as well as methods of programming and numerical calculations on the PC are used..

Results and discussion. Designed by us for a centrifugal mineral fertilizer spreader, the design of a centrifugal scattering working body with an inclined axis of rotation includes a kinematic connected with the drive mechanism in a rotational motion of a flat disk with radially mounted blades on its working surface. In this case, the axis of rotation of the centrifugal 
scattering working body (disk) is located at an angle $\alpha$ to the horizontal plane

For such a centrifugal dispersing working organ of mineral fertilizers, we will construct a calculated mathematical model of the motion of a material particle along its radially located shoulder blade, with the position that the axis of rotation of the disperser disc has a slope. The first step in this will be the construction of an equivalent scheme, in which we will denote the material particle of mineral fertilizer moving along the shoulder of the inclined dispersive disk and show the forces acting on it (Fig. 1). Let's assume that $M-$ initial position of the mineral fertilizer on the shovel, point $S$ - current position of the fertilizer particle on the shoulder blade, point $O$ - center of rotation of the centrifugal scattering working body (disk).

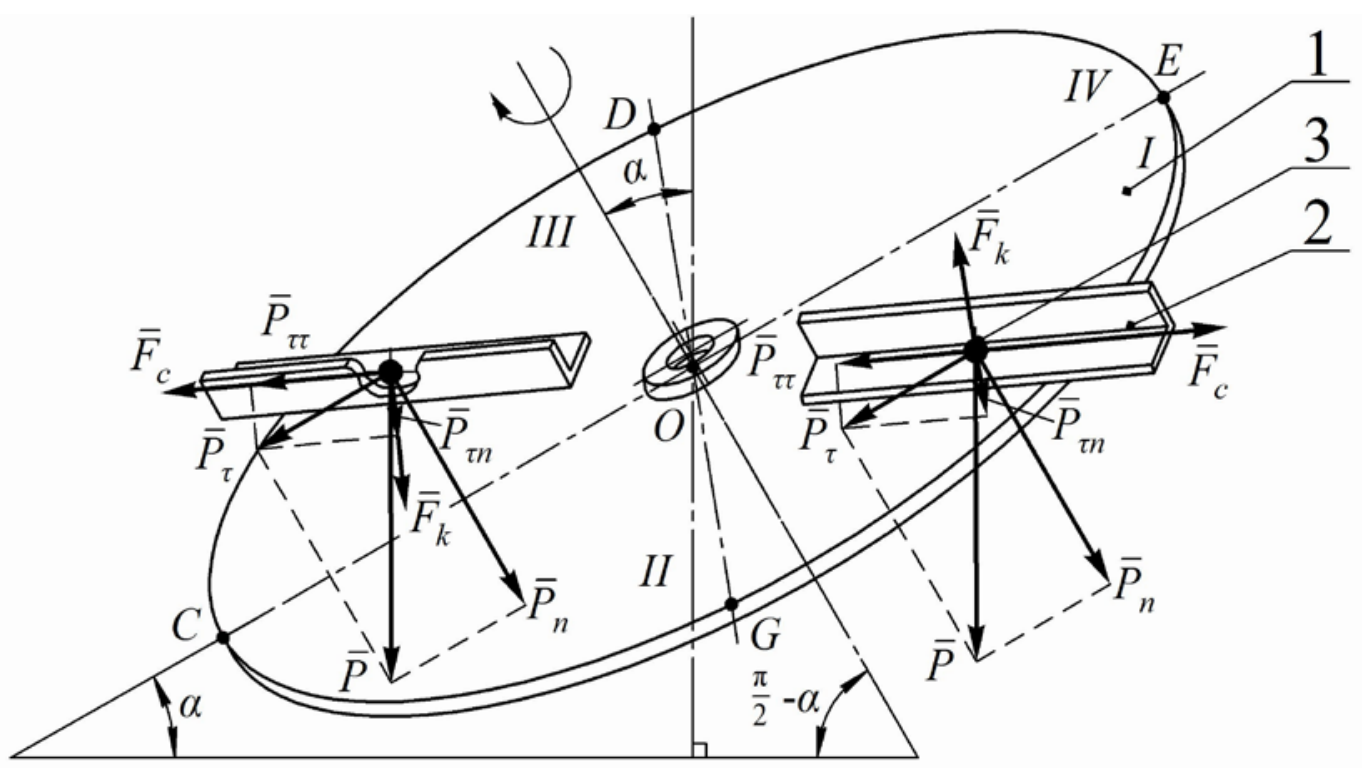

a) 


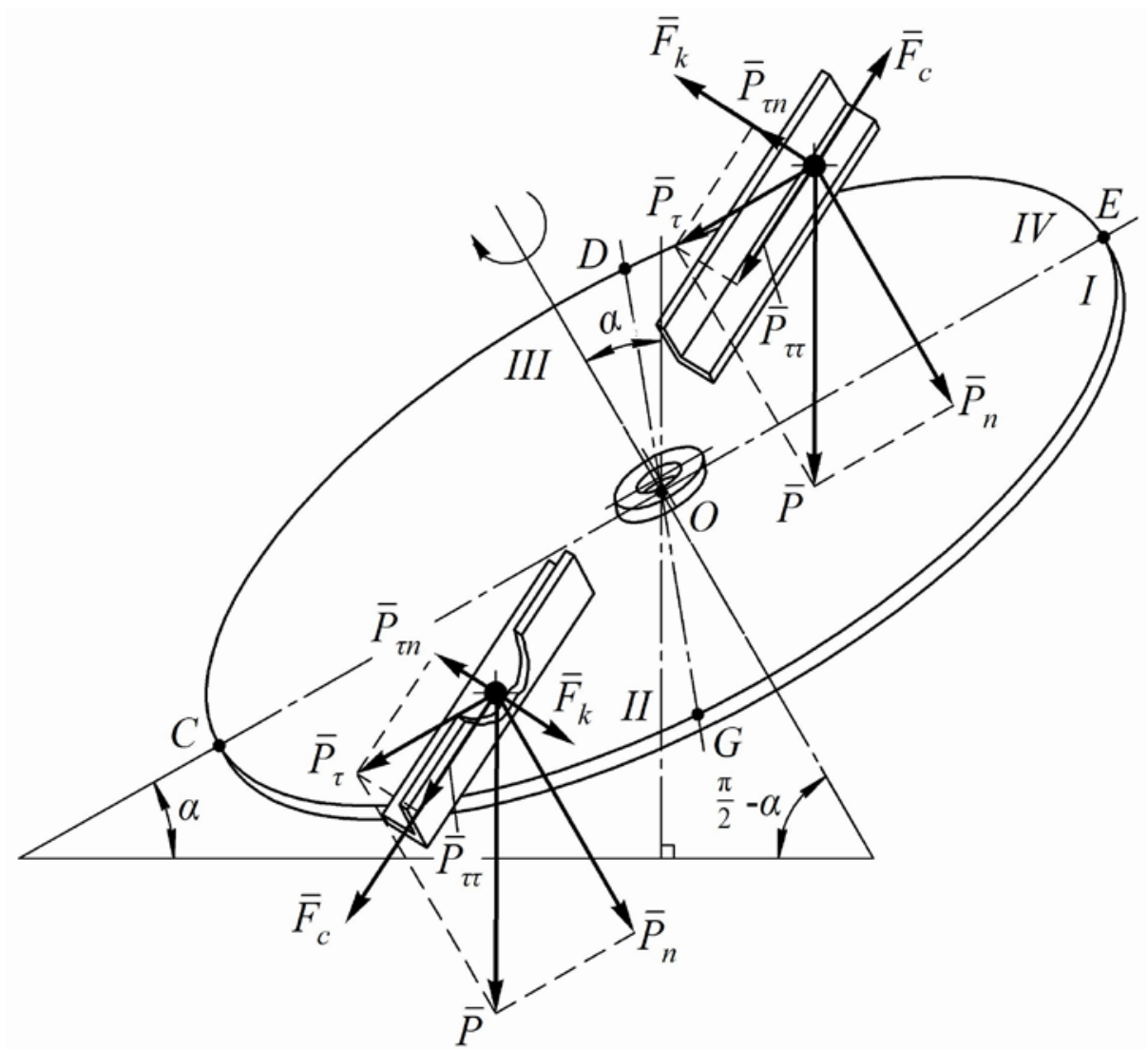

б)

Fig. 1. An equivalent scheme of motion of a particle of mineral fertilizers along the blade of a scattering disc inclined at an angle $\alpha$ to the horizon $(a, b$ - hence the fertilizer particle moves along blades within sectors I, III and II, IV):

1 - disk; 2 - scapula; 3 - fertilizer particle

Immediately, to simplify the analytical solution of this problem, we accept the following assumptions:

- the coefficient of friction of mineral fertilizer particles on the surface of the blade has a constant value;

- the nature of the movement of each particle of fertilizer is the same and corresponds to the nature of the movement of the entire mass of fertilizers along the shoulder blade;

- the fertilizer particle moves along the blade along the segment, which is common to the vertical wall of the shoulder blade and its bottom, without rolling;

- the thickness of the blade and the diameter of the particle of mineral fertilizer despise. 
A rather significant difference between the dispersion of mineral fertilizer particles and the centrifugal dispersive working body with a sloping axis of rotation, in comparison with the horizontally located, is that there are some differences in the location of the vectors applied to the material particle forces, depending on the position of the inclined centrifugal disk And the mineral fertilizers are attracted to the shoulder blades: in the upper part of the inclined disk or in its lower part, in the right of the axis of rotation or in the left part. This circumstance must also be taken into account when analyzing this task.

Since the value of the absolute acceleration rate $V_{A C}$ of the fertilizer particle from the centrifugal scattering working body is the sum of the relative speed $V_{r c}$ of the fertilizer particle during its onset from the surface of the centrifugal dispersal disk $\left(\mathrm{m} \cdot \mathrm{s}^{-1}\right)$ and the portable speed $V_{N C}$ of the fertilizer particle during its onset from the surface of the centrifugal scattering worker the organ $\left(\mathrm{m} \cdot \mathrm{s}^{-1}\right)$, that Is $V_{A C}=\sqrt{V_{r C}^{2}+V_{N C}^{2}}$, the portable speed $V_{N C}$, in turn, is equal to $V_{N C}=\omega R$ where $\omega$ - the angular velocity of the centrifugal scattering working body $\left(\mathrm{c}^{-1}\right)$ and $R$ - the radius of the centrifugal scattering worker organ $(\mathrm{m})$, to determine the speed $V_{A C}$ necessary to first determine the unknown relative speed $V_{r c}$.

Further, due to the fact that the projection of the component of the weight $\bar{P}$ of the fertilizer particle on the segment $A B$ in the process of its motion along the blade changes the direction of the vector, it is advisable to divide the centrifugal scattering working element into sectors in such a way that the direction of the vector during its movement within each the sector has not changed. By completing this, we get four equal sectors: EOG - I; GOC - II; COD - III; DOE - IV (fig. 2). 

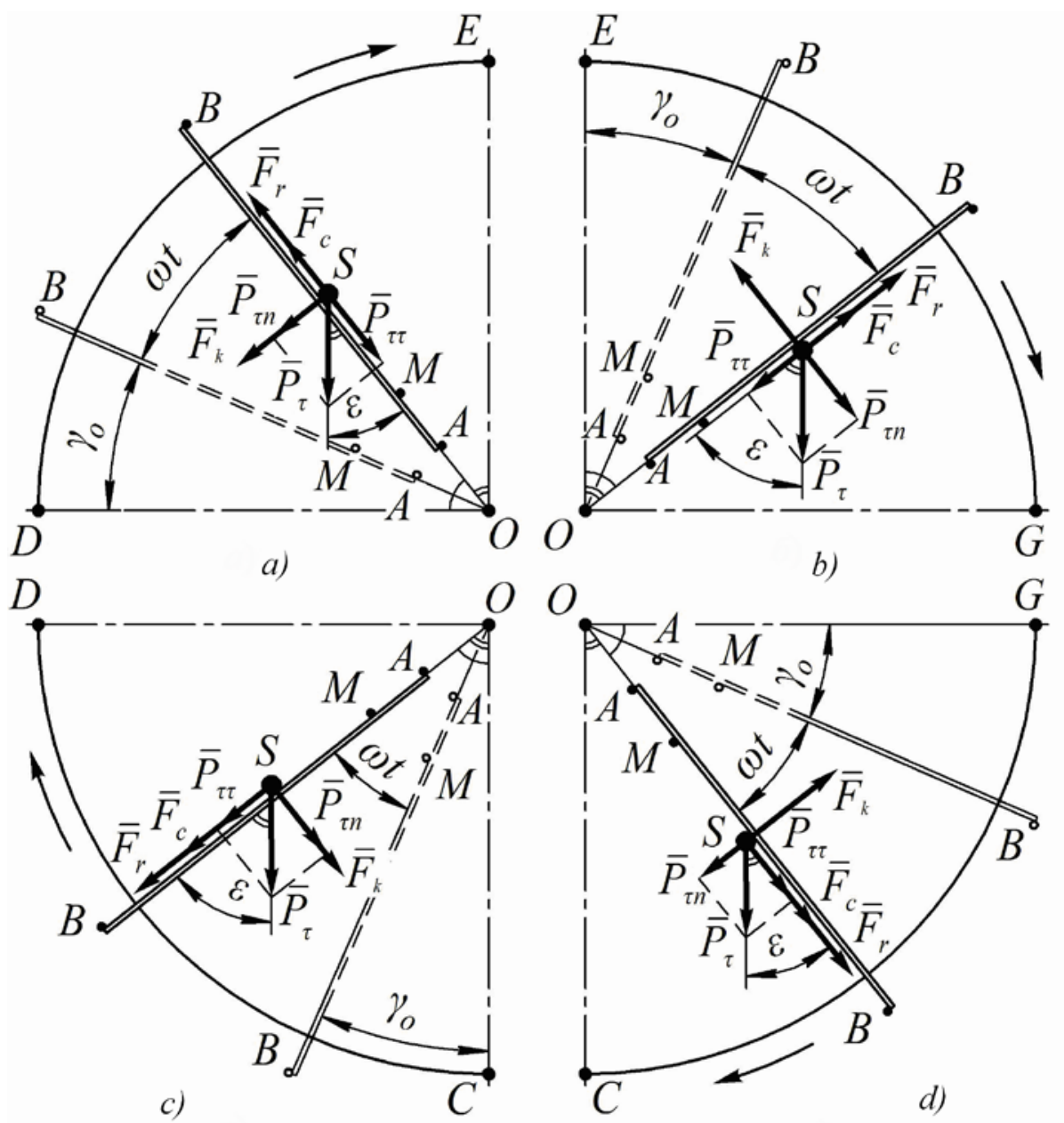

Fig. 2. Scheme for determining the resultant force, under the action of which a mineral fertilizer unit moves along the center of the blade scattering disk:

$a, b, c, d-$ respectively, the fertilizer particle moves along the blade within the sectors IV, I, III and II

The segments $E C$ and $D G$ are mutually perpendicular to the diameters of the flat scattering disk, and the EC segment forms with a horizontal plane of the angle $\alpha$.

Determine the value of the forces acting on the material particle $M$ of mineral fertilizers, and write the equation for determining the resultant force $F_{r}$, under the action of which this particle will move along the shoulder blade (Fig. 1, 2). Since the movement of the material particle $M$ of the mineral fertilizer is carried out in a straight line along the surface of the 
blade, then we write this equation in the form of projections on the axis coinciding with the surface of the shoulder blade itself:

$$
F_{r}=F_{C} \pm P_{\tau \tau}-f_{f} F_{k}-f_{f} P_{n}-f_{f} P_{\tau n},
$$

where $f_{f}$ - the coefficient of friction $M$ of the fertilizer particle on the surface of the shoulder blade.

Determine the value attached to the material particle $M$ forces included in the expression (1).

The resulting force, under the action of which the mineral fertilizer $M$ is moving along the shoulder blade, is defined as follows:

$$
F_{r}=m \frac{d^{2} L}{d t^{2}},
$$

where $m$ - mass of fertilizer particles, $\mathrm{kg} ; L$ - the path that passed the fertilizer particle along the shoulder blade, $\mathrm{m} ; t$ - time of movement of the fertilizer particle along the shoulder blade, $p$.

The centrifugal force $\bar{F}_{c}$ of inertia can be found using this expression:

$$
F_{c}=m r \omega^{2},
$$

where $r$-distance from the center of rotation of the centrifugal dispersal disk to the current position of the fertilizer particle on the shoulder blade, $m$; $\omega$ - angular velocity of the centrifugal dispersal disk, $\mathrm{p}^{-1}$.

The design of the component of the weight $\bar{P}_{\tau}$ of the fertilizer particle on the segment $A B$ is defined as follows:

$$
P_{\tau \tau}=P_{\tau} \cos \varepsilon,
$$

where $\varepsilon$-angle between the component of the force of weight $\bar{P}_{\tau}$ and its projection on the segment $A B$, rad.

The component weight $\bar{P}_{\tau}$ of the fertilizer particle, acting along the disk surface parallel to the segment of the $E C$, will be determined by this expression:

$$
P_{\tau}=P \sin \alpha
$$


where $\alpha$-angle between the rotation axis of the centrifugal dispersal disk and the vertical plane, rad.

The force $\bar{P}$ of the particle will be equal to the fertilizer:

$$
P=m g \text {, }
$$

where $g$ - acceleration of free fall, $\mathrm{m} \cdot \mathrm{s}^{-2}$.

The force of inertia of Coriolis is determined by this expression:

$$
F_{k}=2 m \omega \frac{d L}{d t} .
$$

The component weight of the particle of the fertilizer acting on the normal to the bottom of the shoulder blade, has the following form:

$$
P_{n}=P \cos \alpha \text {. }
$$

The projection of the component of the weight $P_{\tau}$ of the particle of fertilizer on the normal $n$ to the segment $A B$ will equal:

$$
P_{\tau n}=P_{\tau} \sin \varepsilon .
$$

It is worth noting that if the particles (stream) of mineral fertilizers enter the surface of the centrifugal dispersal disk within the sector I or IV, then in equation (4) before force $P_{\tau \tau}$ it is necessary to put the sign "-", and if within the sector II or III, then it is necessary before the given force to put a sign " +".

Substituting the values of the forces due to the expressions (2) - (9) in equation (1), we obtain the differential equation of the motion of the mineral fertilizer particle $M$ along the vanes of the centrifugal dispersion disk inclined at an angle $\alpha$ to the horizon:

$$
m \frac{d^{2} L}{d t^{2}}=m r \omega^{2} \pm m g \sin \alpha \cdot \cos \varepsilon-f_{f}\left(2 m \omega \frac{d L}{d t}+m g \cos \alpha+m g \sin \alpha \cdot \sin \varepsilon\right) .
$$

As we see from Fig. 2, depending on the sector in which the mineral fertilizer will be fed to the surface of the centrifugal dispersal disk, the value of the angle $\varepsilon$ between the composite weight vector $\bar{P}_{\tau}$ and its projection on the segment $A B$ will be different, and the value of the given angle is 
determined by using the following four expressions:

$\varepsilon=\gamma_{o}+\omega t-$ for a case where mineral fertilizers enter the surface of a centrifugal dispersion disk within the sector I,

where $\gamma_{0}$ - angle, formed by segments $O E$ and $O B$, at the moment of contact of a fertilizer particle with a shoulder blade, rad.;

$\varepsilon=\frac{\pi}{2}-\left(\gamma_{o}+\omega t\right)$ - for a case where mineral fertilizers enter the surface of a centrifugal dispersion disk within the sector II, where $\gamma_{o}$ - angle, formed by segments $O G$ and $O B$, at the moment of contact of fertilizer particle with a shoulder blade, rad.;

$\varepsilon=\gamma_{o}+\omega t-$ for a case where mineral fertilizers enter the surface of a centrifugal dispersion disk within the sector III,

where $\gamma_{o}$ - angle formed by segments $O C$ and $O B$, at the moment of contact of fertilizer particle with a shoulder blade, rad.;

$\varepsilon=\frac{\pi}{2}-\left(\gamma_{o}+\omega t\right)$ - for a case where mineral fertilizers enter the surface of a centrifugal dispersion disk within the sector IV, where $\gamma_{0}$ - angle formed by segments $O D$ and $O B$, at the moment of contact of a fertilizer particle with a shoulder blade, rad.

Then write the expression to determine the distance $r$ from the center of rotation of the centrifugal dispersal disk to the current position $S$ of the fertilizer particle on the shovel. It is defined by using this expression

$$
r=r_{o}+L,
$$

where $r_{o}$ - The fertilizer particle supply radius to the centrifugal dispersal disk, $\mathrm{m}$.

Substituting in the expression (10) the value of distance and passing a series of transformations, we get:

$$
\frac{d^{2} L}{d t^{2}}=\omega^{2} r_{o}+\omega^{2} L \pm g \sin \alpha \cdot \cos \varepsilon-2 f_{f} \omega \frac{d L}{d t}-f_{f} g \cos \alpha-f_{f} g \sin \alpha \cdot \sin \varepsilon
$$


Let's consider the case when fertilizers are fed to the surface of a centrifugal dispersion disk within the sector II (GOC). Then, equation (12) will already have such a form:

$$
\begin{aligned}
& \frac{d^{2} L}{d t^{2}}+2 f_{f} \omega \frac{d L}{d t}-\omega^{2} L=\left(\omega^{2} r_{o}-f_{f} g \cos \alpha\right)+ \\
& +g \sin \alpha \cdot \sin \left(\gamma_{o}+\omega t\right)-f_{f} g \sin \alpha \cdot \cos \left(\gamma_{o}+\omega t\right) .
\end{aligned}
$$

Thus, a linear second-order differential equation with constant coefficients and the right-hand side is obtained.

We solve the resulting differential equation (13). His characteristic equation will look like this:

$$
\lambda^{2}+2 f_{f} \omega \lambda-\omega^{2}=0
$$

and its roots will accordingly be equal:

$$
\begin{aligned}
& \lambda_{1}=\omega\left(\sqrt{f_{f}^{2}+1}-f_{f}\right), \\
& \lambda_{2}=\omega\left(-\sqrt{f_{f}^{2}+1}-f_{f}\right) .
\end{aligned}
$$

We write the general solution $\bar{L}$ of equation (13) without the righthand side:

$$
\bar{L}=C_{1} e^{\lambda_{1} t}+C_{2} e^{\lambda_{2} t},
$$

where $C_{1}$ and $C_{2}$ - arbitrary steel.

Here is a partial solution $L^{*}$ equation (13).

Let's introduce the following notation:

$$
\omega^{2} r_{o}-f_{f} g \cos \alpha=K, \quad g \sin \alpha=U .
$$

Then the right side of the differential equation (13) taking into account the accepted notation will have this form:

$$
K+U \sin \left(\gamma_{o}+\omega t\right)-f_{f} U \cos \left(\gamma_{o}+\omega t\right) .
$$

In this case, the partial solution of the nonhomogeneous equation is sought in the following form:

$$
L^{*}=W \sin \left(\gamma_{o}+\omega t\right)+Z \cos \left(\gamma_{o}+\omega t\right)+J,
$$


where $W, Z$ and $J$ - unknown coefficients.

These unknown coefficients are determined by the method of uncertain coefficients. To do this, we differentiate twice the partial solution (19). We have:

$$
\begin{aligned}
& \frac{d L^{*}}{d t}=\omega W \cos \left(\gamma_{o}+\omega t\right)-\omega Z \sin \left(\gamma_{o}+\omega t\right), \\
& \frac{d^{2} L^{*}}{d^{2} t}=-\omega^{2} W \sin \left(\gamma_{o}+\omega t\right)-\omega^{2} Z \cos \left(\gamma_{o}+\omega t\right) .
\end{aligned}
$$

We obtain the expressions (20) and (21) in equation (13). We will have:

$$
\begin{aligned}
& -\omega^{2} W \sin \left(\gamma_{o}+\omega t\right)-\omega^{2} Z \cos \left(\gamma_{o}+\omega t\right)+ \\
& +2 f_{f} \omega\left[\omega W \cos \left(\gamma_{o}+\omega t\right)-\omega Z \sin \left(\gamma_{o}+\omega t\right)\right]- \\
& -\omega^{2}\left[W \sin \left(\gamma_{o}+\omega t\right)+Z \cos \left(\gamma_{o}+\omega t\right)+J\right]= \\
& =K+U \sin \left(\gamma_{o}+\omega t\right)-f_{f} U \cos \left(\gamma_{o}+\omega t\right) .
\end{aligned}
$$

We obtain the expressions (20) and (21) in equation (13). We will have:

$$
\begin{aligned}
& -\omega^{2} W \sin \left(\gamma_{o}+\omega t\right)-\omega^{2} Z \cos \left(\gamma_{o}+\omega t\right)+ \\
& +2 f_{f} \omega^{2} W \cos \left(\gamma_{o}+\omega t\right)-2 f_{f} \omega^{2} Z \sin \left(\gamma_{o}+\omega t\right)- \\
& -\omega^{2} W \sin \left(\gamma_{o}+\omega t\right)-\omega^{2} Z \cos \left(\gamma_{o}+\omega t\right)-\omega^{2} J= \\
& =K+U \sin \left(\gamma_{o}+\omega t\right)-f_{f} U \cos \left(\gamma_{o}+\omega t\right) .
\end{aligned}
$$

Equate the coefficients with the corresponding trigonometric functions. We have:

$$
\left.\begin{array}{l}
-\omega^{2} W-2 f_{f} \omega^{2} Z-\omega^{2} W=U, \\
-\omega^{2} Z+2 f_{f} \omega^{2} W-\omega^{2} Z=-f_{f} U, \\
-\omega^{2} J=K,
\end{array}\right\}
$$

or

$$
\left.\begin{array}{l}
-2 \omega^{2} W-2 f_{f} \omega^{2} Z=U, \\
-2 \omega^{2} Z+2 f_{f} \omega^{2} W=-f_{f} U, \\
-\omega^{2} J=K .
\end{array}\right\}
$$


From the system of linear equations (25) for unknowns $R, S$ and $T$ we find the values of these unknown coefficients. They will be equal:

$$
J=-\frac{K}{\omega^{2}}, \quad Z=0, \quad W=-\frac{U}{2 \omega^{2}} .
$$

Substituting the values of the obtained coefficients (26) in expression (19), we obtain a partial solution of the nonhomogeneous differential equation:

$$
L^{*}=-\frac{U}{2 \omega^{2}} \sin \left(\gamma_{o}+\omega t\right)-\frac{K}{\omega^{2}} .
$$

The general solution of the differential equation (13) can be written as follows:

$$
L=\bar{L}+L^{*}=C_{1} e^{\lambda_{1} t}+C_{2} e^{\lambda_{2} t}-\frac{U}{2 \omega^{2}} \sin \left(\gamma_{o}+\omega t\right)-\frac{K}{\omega^{2}} .
$$

Arbitrary constants $C_{1}$ and $C_{2}$ we find from the following initial conditions:

at $t=0: L=0, \frac{d L}{d t}=0$.

Next, we differentiate according $t$ to the expression (18). We will have:

$$
\frac{d L}{d t}=\lambda_{1} C_{1} e^{\lambda_{1} t}+\lambda_{2} C_{2} e^{\lambda_{2} t}-\frac{U}{2 \omega} \cos \left(\gamma_{o}+\omega t\right) .
$$

Using the above initial conditions, we obtain the following system of algebraic equations for unknowns $C_{1}$ and $C_{2}$ :

$$
\left.\begin{array}{l}
C_{1}+C_{2}-\frac{U}{2 \omega^{2}} \sin \gamma_{o}-\frac{K}{\omega^{2}}=0, \\
\lambda_{1} C_{1}+\lambda_{2} C_{2}-\frac{U}{2 \omega} \cos \gamma_{o}=0 .
\end{array}\right\}
$$

Solving the system of equations (30) we finally find the value of arbitrary constants $C_{1}$ and $C_{2}$ :

$$
C_{1}=\frac{U \cos \gamma_{o}}{2 \omega\left(\lambda_{1}-\lambda_{2}\right)}-\frac{U \lambda_{2} \sin \gamma_{o}}{2 \omega^{2}\left(\lambda_{1}-\lambda_{2}\right)}-\frac{K \lambda_{2}}{\omega^{2}\left(\lambda_{1}-\lambda_{2}\right)},
$$


and

$$
C_{2}=-\frac{U \cos \gamma_{o}}{2 \omega\left(\lambda_{1}-\lambda_{2}\right)}+\frac{U \lambda_{2} \sin \gamma_{o}}{2 \omega^{2}\left(\lambda_{1}-\lambda_{2}\right)}+\frac{K \lambda_{2}}{\omega^{2}\left(\lambda_{1}-\lambda_{2}\right)}+\frac{U \sin \gamma_{o}}{2 \omega^{2}}+\frac{K}{\omega^{2}} .
$$

Substituting the obtained values (31) and (32) of arbitrary constants $C_{1}$ and $C_{2}$ in expression (28), we obtain the final solution of the differential equation (13):

$$
\begin{aligned}
L & =\left[\frac{U \cos \gamma_{o}}{2 \omega\left(\lambda_{1}-\lambda_{2}\right)}-\frac{U \lambda_{2} \sin \gamma_{o}}{2 \omega^{2}\left(\lambda_{1}-\lambda_{2}\right)}-\frac{K \lambda_{2}}{\omega^{2}\left(\lambda_{1}-\lambda_{2}\right)}\right] e^{\lambda_{1} t}+ \\
& +\left[-\frac{U \cos \gamma_{o}}{2 \omega\left(\lambda_{1}-\lambda_{2}\right)}+\frac{U \lambda_{2} \sin \gamma_{o}}{2 \omega^{2}\left(\lambda_{1}-\lambda_{2}\right)}+\frac{K \lambda_{2}}{\omega^{2}\left(\lambda_{1}-\lambda_{2}\right)}+\right. \\
& \left.+\frac{U \sin \gamma_{o}}{2 \omega^{2}}+\frac{K}{\omega^{2}}\right] e^{\lambda_{2} t}-\frac{U \sin \left(\gamma_{o}+\omega t\right)}{2 \omega^{2}}-\frac{K}{\omega^{2}} .
\end{aligned}
$$

Expression (33) stipulates the law of displacement of a material particle of mineral fertilizers along the vanes of a centrifugal dispersion disk inclined at an angle $\alpha$ to the horizon.

Substituting expressions (31) and (32) in expression (29), we obtain the law of changing the rate of relative movement $V_{r}$ of the mineral fertilizer along the blade at an arbitrary time $t$ :

$$
\begin{aligned}
V_{r} & =\frac{d L}{d t}=\left[\frac{U \cos \gamma_{o}}{2 \omega\left(\lambda_{1}-\lambda_{2}\right)}-\frac{U \lambda_{2} \sin \gamma_{o}}{2 \omega^{2}\left(\lambda_{1}-\lambda_{2}\right)}-\frac{K \lambda_{2}}{\omega^{2}\left(\lambda_{1}-\lambda_{2}\right)}\right] \lambda_{1} e^{\lambda_{1} t}+ \\
& +\left[-\frac{U \cos \gamma_{o}}{2 \omega\left(\lambda_{1}-\lambda_{2}\right)}+\frac{U \lambda_{2} \sin \gamma_{o}}{2 \omega^{2}\left(\lambda_{1}-\lambda_{2}\right)}+\frac{K \lambda_{2}}{\omega^{2}\left(\lambda_{1}-\lambda_{2}\right)}+\right. \\
& \left.+\frac{U \sin \gamma_{o}}{2 \omega^{2}}+\frac{K}{\omega^{2}}\right] \lambda_{2} e^{\lambda_{2} t}-\frac{U}{2 \omega} \cos \left(\gamma_{o}+\omega t\right) .
\end{aligned}
$$

To determine the time $t_{1}$ of movement of a mineral fertilizer particle along the blade from its point of delivery (point $M$ ) to its point of descent from the shoulder blade (point $B$ ), it is necessary in expression (35) instead $L$ of setting its value $L=R-r_{o}$, which determines the distance 
between the points $M$ and $B$, and to solve the equation with respect to time $t_{1}$. Substituting the received value of time $t_{1}$ into equation (34), we obtain the value of the relative speed $V_{r c}$ of the motion of the fertilizer fraction at the time of its ascent from the surface of the disperse disk.

Thus, given that $V_{A C}=\sqrt{V_{r C}^{2}+V_{N C}^{2}}$ we have the ability to determine the magnitude of the absolute velocity $V_{A C}$ at the time of its ascent from the surface of the disperse disk, when the mineral fertilizers are fed to the surface of the centrifugal dispersal disk within the sector II (GOC).

Using the obtained analytical expressions, in accordance with the developed program, numerical calculations were made on the PC, which made it possible to determine the influence of parameters $\omega, r_{o}$ and $\alpha$ on $V_{A C}$.

It was established that the increase $\alpha$ from 0 ㅇ to 90 leads to change $V_{A C}$ no more than $0,1 \mathrm{~m} \cdot \mathrm{s}^{-1}$. Influence $\omega$ and $r_{o}$ on $V_{A C}$ shown on (Fig. 3).

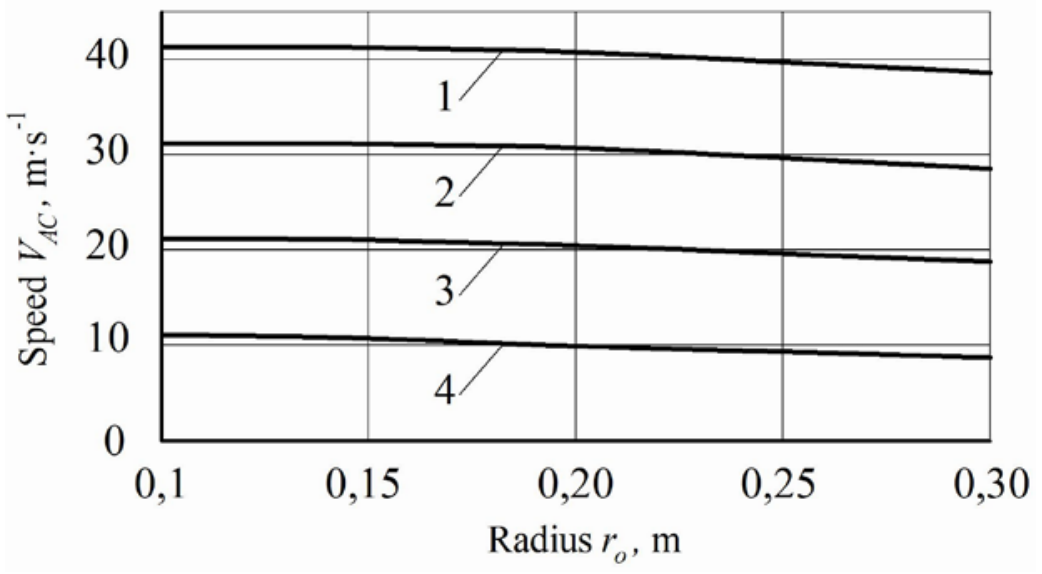

Fig. 3. Dependence of the absolute speed $V_{A C}$ of the particle's fertilizer from the radius $r_{o}$ of its filing (fertilizers are fed to the disk surface in sector II, with $\left.R=0,34 \mathrm{~m}, f_{f}=0,55, \alpha=30^{\circ}, \gamma_{o}=1^{\circ}\right)$ : $1-\omega=104,6 \mathrm{~s}^{-1} ; 2-\omega=78,5 \mathrm{~s}^{-1} ; 3-\omega=52,3 \mathrm{~s}^{-1} ; 4-\omega=26,2 \mathrm{cs}^{-1}$.

As can be seen from the charts of Fig. 3, at $\omega=104,6 \mathrm{~s}^{-1}$ increase $r_{o}$ from $0,1 \mathrm{~m}$ to $0,3 \mathrm{~m}$ leads to a decrease $V_{A C}$ from $41,32 \mathrm{~m} \cdot \mathrm{s}^{-1}$ to $38,21 \mathrm{~m} \cdot \mathrm{s}^{-1}$. 
In addition, an increase $\omega$ from $26,2 \mathrm{~s}^{-1}$ to $104,6 \mathrm{~s}^{-1}$ at $r_{o}=0,1 \mathrm{~m}$ leads to an increase $V_{A C}$ from $10,33 \mathrm{~m} \cdot \mathrm{s}^{-1}$ to $41,32 \mathrm{~m} \cdot \mathrm{s}^{-1}$.

The choice of the sector for the supply of mineral fertilizers to the scattering disc slightly affects the value $V_{A C}$. Thus, when $R=0,34 \mathrm{~m}$, $f_{f}=0,55, \alpha=90^{\circ}, r_{o}=0,2 \mathrm{~m}, \gamma_{o}=1^{o}, \omega=104,6 \mathrm{~s}^{-1}$ value $V_{A C}$ will be: sector I $V_{A C}=40,74 \mathrm{~m} \cdot \mathrm{s}^{-1} ;$ sector II $-V_{A C}=40,78 \mathrm{~m} \cdot \mathrm{s}^{-1} ;$ sector III $-V_{A C}=40,75 \mathrm{~m} \cdot \mathrm{s}^{-1}$; sector IV $-V_{A C}=40,74 \mathrm{~m} \cdot \mathrm{s}^{-1}$.

To determine the angle of the fertilizer from the centrifugal dispersal disk, you must first determine their place of the east. In view of the fact that the position of the blade at the time of contact with the proportion of fertilizers is known, it is appropriate to use to determine the location of fertilizer from the dispersal disk angle $\beta_{a}$ their overclocking The angle of acceleration is the angle between the positions of the shoulder blade at the time of contact with the fertilizer and the same blade at the time of the commencement of fertilizer from it.

If the angle $\beta_{a}$ value is equal to $\beta_{a}=\omega t:$, then using the expressions (33), (34) and equation (35) it is possible to investigate the effect of the parameters $\omega, r_{o}$ and $\alpha$ on this angle $\beta_{a}$. Based on the numerical calculations performed on the PC, the graphical results shown in Fig. 4 and 5.

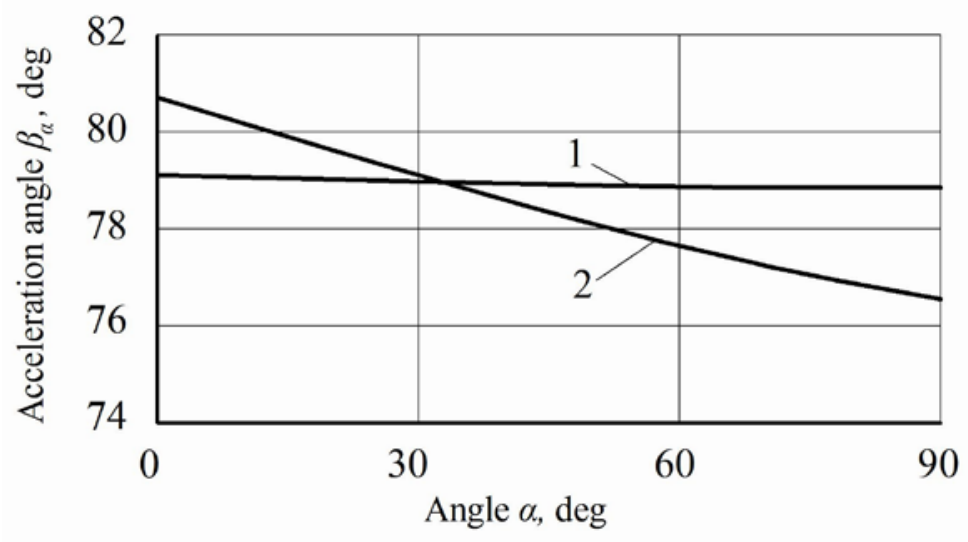


Fig. 4. Dependence of the angle $\beta_{a}$ of dispersal of a particle of mineral fertilizers

from the angle $\alpha$ (fertilizers are fed to the disk surface in the sector II, at $\left.R=0,34 \mathrm{~m}, f_{f}=0,55, r_{o}=0,2 \mathrm{~m}, \gamma_{o}=1^{\circ}\right)$ :

$$
1-\omega=104,6 \mathrm{~s}^{-1} ; 2-\omega=26,2 \mathrm{~s}^{-1} \text {. }
$$

Based on charts of rice. 4 it can be concluded that the effect of the angle $\alpha$ of inclination of the axis of rotation of the centrifugal dispersal disk on the angle $\beta_{a}$ of acceleration of the particle of fertilizers, at high values $\omega$, is insignificant. So, at $\omega=104,6 \mathrm{~s}^{-1}$ an increase $\alpha$ from 0 to $90^{\circ}$ leads to a decrease $\beta_{a}$ from $79,13^{\circ}$ to a total $78,86^{\circ}$.

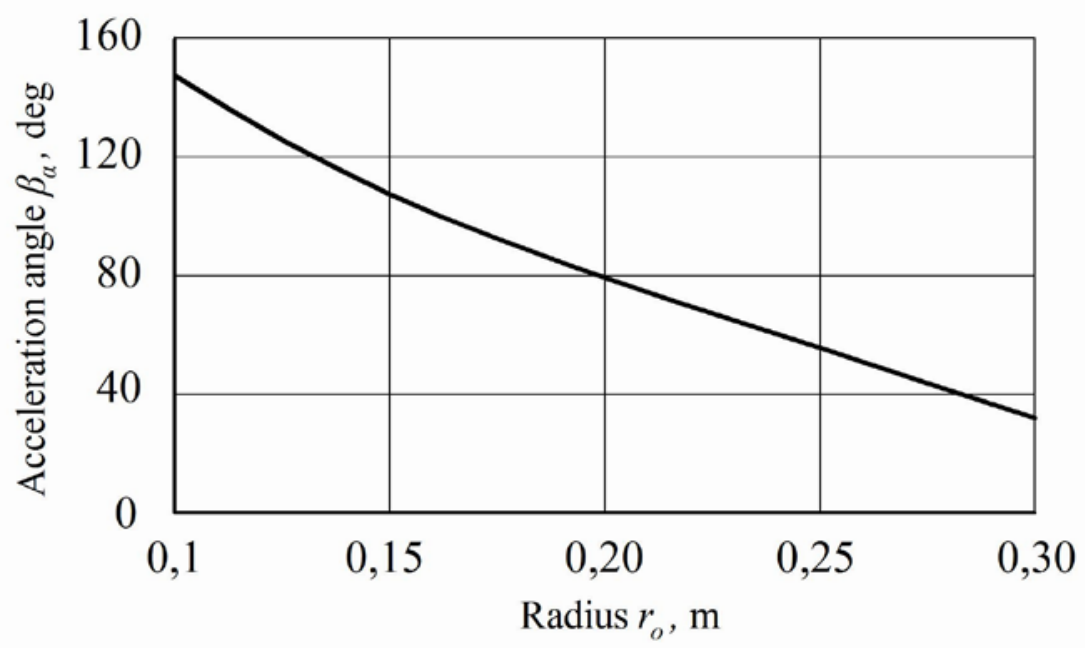

Fig. 5. Dependence of the angle $\beta_{a}$ of dispersal of a particle of mineral fertilizers

from the radius $r_{o}$ of its filing to the disk (fertilizers are fed to the disk surface in sector II $R=0,34 \mathrm{~m}, f_{f}=0,55, \omega=104,6 \mathrm{~s}^{-1}, \alpha=30^{\circ}, \gamma_{o}=1^{o}$ )

Established that increase $r_{o}$ from $0,1 \mathrm{~m}$ to $0,3 \mathrm{~m}$ leads to a decrease $\beta_{a}$ from $147,53^{\circ}$ to $32,24^{\circ}$. In addition, the choice of the sector to feed fertilizer on the surface of the disk slightly affects the magnitude of the angle $\beta_{a}$. Thus, when $R=0,34 \mathrm{~m}, f_{f}=0,55, \alpha=90^{\circ}, r_{o}=0,2 \mathrm{~m}, \gamma_{o}=1^{\circ}$, $\omega=104,6 \mathrm{~s}^{-1}$ value $\beta_{a}$ will be: sector I $-\beta_{a}=79,16^{\circ}$; sector II $-\beta_{a}=78,86^{\circ}$; sector III $-\beta_{a}=79,09^{\circ}$; sector IV $-\beta_{a}=79,23^{\circ}$. 
The use of the obtained dependencies and the methodology for determining the angle of the fraction of fertilizers from the surface of the scattering disk enables to obtain the initial data for the calculation of the flight range of fertilizer particles when scattered by a centrifugal dispersal disk. Determining the spreading distance of the fertilizer fraction of the centrifugal scattering working body will, in turn, give the opportunity to justify other rational parameters and operating modes of the centrifugal scattering working body.

\section{Conclusions.}

1. Theoretically obtained new dependences describing the motion of a mineral fertilizer particle along a radially mounted blade of a centrifugal scattering working body whose axis of rotation is located at an angle $\alpha$ to the horizontal plane. In particular, the absolute velocity of the mineral fertilizer fraction is determined at the time of its ascent from the centrifugal scattering working body.

2. It was established that an increase in the value of the angle between the rotation axis of the centrifugal scattering working body and the vertical plane $\alpha$ from $0^{\circ}$ to $90^{\circ}$ changes the value of the absolute speed of the going $V_{A C}$ fertilizer particles from the centrifugal scattering working body no more than $0,1 \mathrm{~m} \cdot \mathrm{s}^{-1}$. Increase of the fertilizer supply radius to the centrifugal scattering working body $r_{o}$, from $0,1 \mathrm{~m}$ to $0,3 \mathrm{~m}$ at the angular velocity of the centrifugal scattering working body $\omega=104,6 \mathrm{~s}^{-1}$ leads to a decrease $V_{A C}$ from $41,32 \mathrm{~m} \cdot \mathrm{s}^{-1}$ to $38,21 \mathrm{~m} \cdot \mathrm{s}^{-1}$. In addition, an increase $\omega$ from $26,2 \mathrm{~s}^{-1}$ to $104,6 \mathrm{~s}^{-1}$ at $r_{o}=0,1 \mathrm{~m}$ leads to an increase $V_{A C}$ from $10,33 \mathrm{~m} \cdot \mathrm{s}^{-1}$ to $41,32 \mathrm{~m} \cdot \mathrm{s}^{-1}$. The choice of the sector for feeding fertilizers to the surface of the centrifugal scattering worker slightly affects the value $V_{A C}$.

3. It was established that the influence on the angle $\alpha$ of dispersal of the fraction of fertilizers $\beta_{a}$, at high values $\omega$, is insignificant. So, with $\omega=104,6 \mathrm{~s}^{-1}$ increase $\alpha$ from $0^{\circ}$ to $90^{\circ}$, it reduces $\beta_{a}$ from $79.13^{\circ}$ to 
78.86\%. In addition, an increase $r_{o}$ from $0.1 \mathrm{~m}$ to $0.3 \mathrm{~m}$ leads to a decrease $\beta_{a}$ from $147.53^{\circ}$ to $32.24^{\circ}$. The choice of the sector for feeding fertilizers to the surface of the centrifugal scattering working body also slightly affects the value $\beta_{a}$.

\section{Bibliography}

1. Adamchuk V.V. The influence of parameters and operating modes of the scattering organ on the movement of mineral fertilizer particles//Bulletin of Agrarian Science. - 2004, №12. - P. 42-45.

2. Bulgakov V., Adamchuk O., Ivanovs S. Theoretical investigations of mineral fertiliser distribution by means of an inclined centrifugal tool. Proceeding of $6^{\text {th }}$ International Conference on Trends in Agricultural Engineering 2016. - 7-9 September 2016. - Prague, Czech Republic. p.p. $109-116$.

3. Biocca M. et al.: Aerodynamic properties of six organomineral fertiliser particles. In: Journal of Agricultural Engineering. No 44 (83), 2013: pp. 411-414.

4. Khomenko Yu.V., Shmelev A.I. Analysis of the designs of foreign disk dispersing apparatuses for the introduction of mineral fertilizers/Collection of scientific works VISKHOM. Directions of research and development of machines for introducing mineral fertilizers into the soil. - Moscow: VISKHOM, 1983. - P. 63-66.

5. Yakimov Y.I., Voloshin N.I., Karabanitsky A.P. and others. Modernization of mineral fertilizer spreaders MVU-5 and MVU-5A//Tractors and agricultural machinery. - 1990, № 7. - P. 27-29.

6. Yakimov Y.I., Karabanitsky A.P. To the design of a place for feeding fertilizers to a centrifugal disk//Proceedings of the Kuban Agricultural Institute. Perfection of agricultural machinery designs. - Krasnodar: Kuban Agricultural Institute, 1976. - Issue 136 (164). - P. 10-15.

7. Vasilenko P.M. The theory of particle motion over rough surfaces of agricultural machines. - Kiev: UAAS, 1960. - 283 p. 
8. Vasilenko P.M. Introduction to agricultural mechanics. - Kiev: Selkhozobrazovanie, 1996. - 252 p.

9. Babukha G.L., Shriber A.A. Interaction of particles of polydisperse material in two-phase streams. - K .: Naukova dumka, 1972. - 175 p.

10. Vasilenko P.M. On the equations of transporting particles in resisting media//Reports of the VASKhNIL. - 1970, № 4. - P. 44-46.

11. Volkov E.A. Numerical methods. - Moscow: Nauka, 1982. - 356 p. 